\title{
Buckling and vibration of thin, flat-walled structures continuous over several spans
}

\author{
Y. K. CHEUNG \& C. DELCOURT
}

\section{Professor F. W. Williams, University of Wales Institute of Science and Technology}

Paragraphs 6-18 of the Paper are devoted to finding the continuous beam eigenvalues and eigenfunctions. This seems unnecessary because a continuous beam is a very simple plane frame and the free vibration of such frames is extensively treated in the literature. Thus equation (9) is the no-sway member equation, with $\alpha$ and $\gamma$ commonly being denoted by $(E I / l) S$ and $(E I / l) S C$, where $S$ and $S C$ are analogous to the well-known stability functions $s$ and sc. Moreover, equation (11) is merely the stiffness matrix formulation of the simple plane frame which results from connecting two such members together to form a continuous beam. However, it is known that a structure can have natural frequencies in addition to those for which the determinant of its stiffness matrix is zero when, as in the Paper, the matrix coefficients are transcendental functions of $\mu_{n}$. The existence of such frequencies is discussed in reference 24 , which also gives a theoretically proven method which is guaranteed to find these and all other natural frequencies of a structure. The method has been extended to buckling problems. ${ }^{25}$ Reference 26 gives a complete description of how the method can be used to find the natural frequencies of a plane frame.

31. Unfortunately, the Authors' method ( $\$ 15)$ only finds those natural frequencies for which the determinant of the stiffness matrix (i.e. the matrix of equation (11) in their example) is zero. Therefore it seems that the Authors should adopt the method described in $\S 30$ (which also overcomes the problem mentioned at the end of $\S 15$ ) or an alternative, as otherwise eigenvalues will be missed for some problems.

32. The danger of missing eigenvalues does not arise for most problems, but is present for any beam with identical spans and clamped ends. Physical argument shows that such a beam will have natural frequencies coinciding with those of a single span with its ends clamped, and that the corresponding modes will involve zero rotations at the intermediate supports. Figs $\mathbf{3 ( b )}, \mathbf{3 ( d )}$ and $3(\mathrm{f})$ illustrate this situation for a beam with two identical spans. Moreover, the first six eigenvalues must clearly correspond to modes 1-6 in Fig. 3. Hence the method in the Paper will miss alternate eigenvalues by finding all those with anti-symmetric modes and missing all those with symmetric modes, because each symmetric mode corresponds to a non-trivial solution of an equation similar to equation (11) for which the vector of the $\theta \mathrm{s}$ is null. Specifically, equation (11) applies to the problem of Fig. 3 when $l_{2}=l_{1}$ and $\theta_{1 n}(0)=\theta_{2 n}\left(l_{2}\right)=0$. Equation (11) can then be simplified to the scalar form

$$
2 \alpha\left(l_{1}\right) \theta_{2 n}(0)=0
$$

and equating the appropriate determinant to zero gives

$$
\alpha\left(l_{1}\right)=0
$$

Paper published: Proc. Instn Civ. Engrs, Part 2, 1977, 63, Mar., 93-103. 


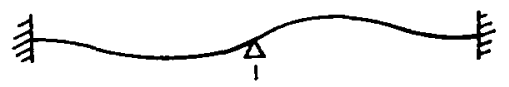

(a)

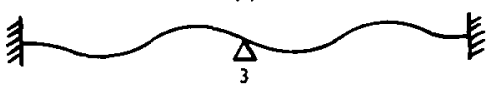

(c)

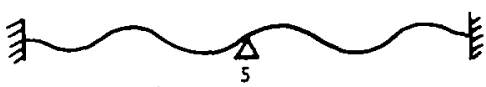

(e)

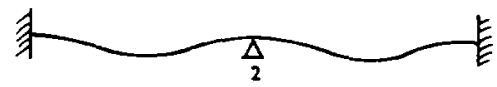

(b)

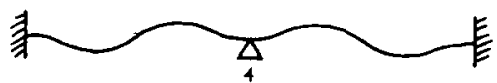

(d)

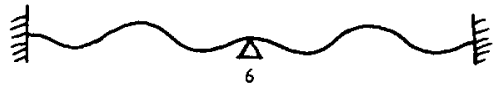

(f)

Fig. 3. Vibration modes for a beam with two identical spans and clamped ends

or, using equation (9)

$$
s_{1} c_{2}-s_{2} c_{1}=0
$$

It can be shown that equation (20) gives the anti-symmetric modes of Fig. 3; the symmetric modes are given by

$$
1-c_{1} c_{2}=0
$$

It follows, from the definition of $\alpha$ in equation (9), that $\alpha\left(l_{1}\right)=\infty$ when equation (21) is satisfied. Hence the symmetric modes correspond to the determinant of the stiffness matrix, which is equal to $2 \alpha\left(l_{1}\right)$, passing through infinity instead of zero.

33. The Authors give the lowest natural frequency (Table 1, case 6) and the lowest critical load (Table 2, case 5) for problems related to Fig. 3. Have they tried to find the second eigenvalues? If so, were the results obtained in error, due to the omission of all symmetric continuous beam eigenfunctions from the set of eigenvalues used?

34. The Authors clearly envisage the finite strip method being used to find all the possible buckling or vibration modes of a plate assembly. The inclusion of modes involving several longitudinal half waves between supports (e.g. the local mode of Fig. 2(b)) is presumably the only reason why $n$ has to be large. However, such modes can be found efficiently by classical methods ${ }^{2-5.12}$ as the assumption of sinusoidal variation of displacement in the longitudinal direction causes negligible errors when the support conditions are incompatible with such variation, because of the relative remoteness of the supports. Indeed, these errors seem likely to be small compared with those resulting from the finite strip analysis, in view of the displacement function assumptions of equation (1), the need to keep $n$ and the number of strips as low as possible, and the possible simplification of the problem, represented by the replacement of the crosssection of Fig. 2(a) by that of Fig. 2(c).

35 . The classical method requires only 12 degrees of freedom to handle the crosssection of Fig. 2(c) using the method given in the appendix of reference 27, and 36 degrees of freedom to handle the cross-section of Fig. 2(a) using a sub-structure approach similar to that given in Fig. 7(c) of reference 27. Furthermore, the associated stiffness matrices have a band width (number of terms within the band and to the right of the leading diagonal) of 7 which is maintained throughout the computation. Increasing $l$ and $S$ in Fig. 2 will not alter these quantities, whereas the comparable finite strip quantities of Table 3 are increased. However, it has to be admitted that the classical method would involve repeating the stiffness matrix calculations. For instance, about 12 repetitions would be required, at each of three assumed half wavelengths of the sinusoidal longitudinal displacement pattern, to obtain the local mode eigenvalue for the problem of Fig. 2. Nevertheless the solution would be obtained rapidly, because of the small number of degrees of freedom and the narrow band width, and it seems certain that the Authors' method must take much longer.

36. A wise policy might be to use classical methods to find the eigenvalues for which 
there are several half waves between supports and the finite strip method, with one strip per plate and a low value of $n$ to reduce the number of degrees of freedom, to find the other eigenvalues.

\section{Dr Cheung and Mrs Delcourt}

In $\$ 30-33$ Professor Williams points out that, in the particular case of a multispan beam with both ends clamped and equal spans, the eigenfrequencies corresponding to a zero displacement vector are missing. We fully agree. This was effectively detected soon after submission of the Paper. Since, in this case, the structure degenerates into substructures which are the different spans clamped, these eigenfrequencies can be easily determined ${ }^{28}$ and introduced where they need to be in the growing sequence of $\mu_{n}$ obtained by finding the zeros of the determinant of the stiffness matrix. The method suggested in reference 24 could effectively be applied to check the number of eigenvalues below each $\mu_{n}$ and then the missing eigenvalues corresponding to a zero displacement vector could be inserted. This is unnecessary in all the cases where one end at least is not clamped, because the solution corresponding to a zero displacement vector is the trivial solution; consequently it is not worthwhile increasing the computer time by calculating roots which will not be used, as the only eigenfrequencies will be those obtained by the method presented in the Paper.

38. We are aware that $\alpha$ and $\beta$ are equivalent to $(E I / l) S$ and $(E I / l) S C$, but the method in the Paper gives a quick and automatic way of determining the eigenfunctions $Y_{n}$ necessary for the derivation of a multispan finite strip having any boundary conditions, number of spans, length of spans and so on.

39. With reference to $\$ 34$ and 35 , it was stated in the Paper that $n$ is proportional to $\alpha(\$ 27), \alpha$ being the number of half-waves for local buckling; it is then natural that $n$ has to be large if $\alpha$ is large.

40. We do not deny the efficiency of the classical methods. First, we compare two numerical methods-finite element and finite strip-but we do not present any comparison between the efficiency of an exact and an approximate method. Second, the influence of the supports can be important in the case of short structures, and in the case of an overall buckling mode which will appear automatically in the present method as well as a local or torsional buckling mode, as no assumption is made about the type of buckling. Furthermore, the present method can easily handle orthotropic structures.

41. As regards $\S 36$, the ultimate aim of the finite strip method applied to multispan structures being the stability analysis and static analysis of multispan flat walled structures, the number $n$ has to be held large enough in view of

(a) predicting an eventual local buckling mode in a stability analysis

(b) approaching accurately the displacement and load distributions in a static analysis.

However, in dynamic analysis, the policy of using a low value of $n$ to find the other eigenvalues without involving too many degrees of freedom can be adopted.

42. Whenever a new approach is presented it is the privilege of the user to combine the advantageous features of the new and the old methods.

\section{References}

24. Wittrick W. H. and Williams F. W. A general algorithm for computing natural frequencies of elastic structures. Q. Jl Mech. Appl. Math., 1971, 24, Part 3, Aug., 263-284.

25. WitTrick W. H. and Williams F. W. An algorithm for computing critical buckling loads of elastic structures. J. Struct. Mech., 1973, 1, No. 4, 497-518.

26. Williams F. W. and WitrRick W. H. An automatic computational procedure for calculating natural frequencies of skeletal structures. Int. J. Mech. Sci., 1970, 12, 781-791. 
DISCUSSION

27. Williams F. W. Computation of natural frequencies and initial buckling stresses of prismatic plate assemblies. J. Sound \& Vibrn, 1972, 21, 87-106.

28. Cheung Y. K. Finite strip method analysis of elastic slabs. J. Engng Mech. Div. Am. Soc. Civ. Engrs, 1968, 94, EM6, Dec., 1365-1378.

\section{Errata}

The last displacement function of equations (1) should read

$$
\begin{aligned}
w= & \sum_{n=1}^{r}\left[\left(1-3 x^{2} / b^{2}+2 x^{3} / b^{3}\right) w_{i n}+\left(x-2 x^{2} / b+x^{3} / b^{2}\right) \theta_{i n}\right. \\
& \left.+\left(3 x^{2} / b^{2}-2 x^{3} / b^{3}\right) w_{f n}+\left(x^{3} / b^{2}-x^{2} / b\right) \theta_{j n}\right] \sum_{s=1}^{S} Y_{s n}
\end{aligned}
$$

44. Equation (4) should read

$$
\frac{\mathrm{d}^{4} Y_{s}}{\mathrm{~d} y_{s}{ }^{4}}-\frac{m_{s} \omega^{2}}{E I_{s}} Y_{s}=0
$$

45. The expression for $C_{s}$ in the penultimate line of $\$ 7$ should read

$$
C_{s}=\left(m_{s} / E I_{s}\right)^{1 / 4}(m / E I)^{-1 / 4}
$$

46. The term $(2,1)$ of the matrix of equation (7) should read

$$
\beta\left(1+s_{1} s_{2}-c_{1} c_{2}\right)
$$

